International Journal of Modern Physics A

(C) World Scientific Publishing Company

\title{
MESON PRODUCTION AT COSY-TOF AND COSY-ANKE
}

\author{
Colin Wilkin \\ Physics and Astronomy Department, UCL, Gower St., London WC1E 6BT, U.K. \\ cw@hep.ucl.ac.uk
}

Received Day Month Year

The roles of the COSY-TOF and COSY-ANKE spectrometers in the measurement of strange meson production are briefly reviewed, mainly in connection with new results on the $p p \rightarrow K^{+} p \Lambda, p p \rightarrow K^{+} p \Sigma^{0}$ and $p p \rightarrow K^{+} n \Sigma^{+}$reactions.

Keywords: Strange mesons; hyperons; cusps.

PACS numbers: 14.40.Aq, 14.20.Jn, 13.75.-n

\section{Introduction}

The COSY-TOF and COSY-ANKE facilities are different in almost every respect. The Time-of-Flight spectrometer ${ }^{1}$ sits on an external beam line and its barrel has a tremendously large acceptance. It relies for its success on measuring the velocities of many particles, with the possibility of afterwards doing kinematic fits. It is especially useful for neutral strange particle production because the time-delayed vertex, from say $\Lambda \rightarrow p \pi^{-}$, can be shown to come from a position downstream of the target.

$\mathrm{ANKE}^{2}$, on the other hand, is a magnetic spectrometer situated at an internal target station of the circulating COSY beam. It can measure the momenta of a variety of positive and negative ejectiles, in particular $K^{+}$and "spectator" protons. However, the overall acceptance is generally very small and often only tiny bits of phase space are sampled. Unlike TOF, it can be used to perform inclusive or semi-inclusive experiments.

Despite their very different characteristics, both ANKE and TOF are well suited for strange particle studies and we shall here concentrate on these. In their seminal 1955 book on particle physics ${ }^{3}$, Bethe and de Hoffmann called the $\Lambda$ and $\Sigma$ baryons, as well as the $\theta$ and $\tau$ mesons, "curious" particles. It is unfortunate that this nomenclature did not stick or we could have been saying that the $K^{+}$had curiosity value +1 and talk about "associated curiosity production"!

\section{The $p p \rightarrow K^{+} p \Lambda$ reaction}

The most detailed measurements of differential observables in the exclusive $p p \rightarrow$

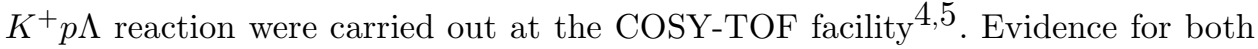


the importance of the $\Lambda p$ final state interaction (FSI) and the $N^{*}(1650)$ isobar is clear from the Dalitz plots obtained at 2.95, 3.20, and $3.30 \mathrm{GeV} / c$. Angular distributions in the overall c.m. frame show significant anisotropy for the $\Lambda$ and proton, whereas the $K^{+}$is fairly flat. The combined data set will provide guidance in the construction of theoretical models but let me concentrate here on just one aspect, viz. the behavior of the $K^{+}$missing-mass spectra in the vicinity of the $\Sigma N$ thresholds.

Using the isotropy in the $K^{+}$production angle ${ }^{5}$, we show in Fig. 1 the forward $K^{+}$differential cross section deduced from the COSY-TOF data 4 . For all beam momenta there is an enhancement at the $\Sigma N$ threshold that must be due to the $\Sigma N \rightarrow \Lambda p$ channel coupling. This cusp effect becomes more pronounced as the beam momentum is lowered and this might be due to the overlap of the $N^{*}(1650)$ with the cusp region then representing a larger fraction of the available phase space.

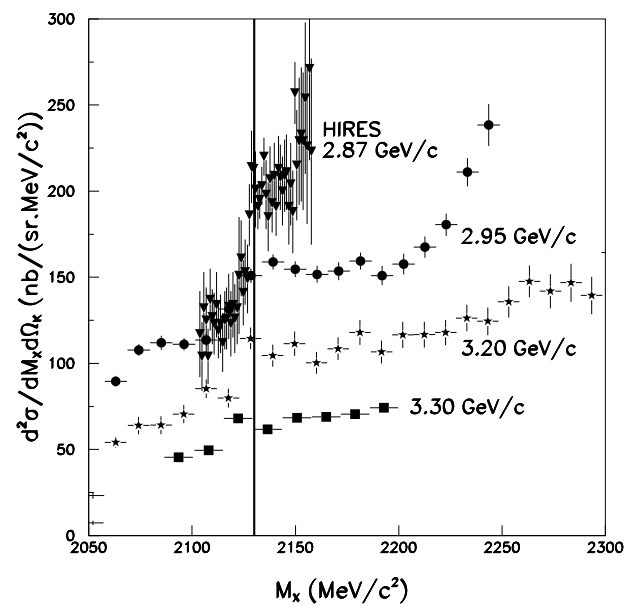

Fig. 1. Forward inclusive cross section for $p p \rightarrow K^{+} X$ as a function of the missing-mass $M_{X}$. The COSY-TOF data at $2.95,3.20$, and $3.30 \mathrm{GeV} / c$ were derived from exclusive $p p \rightarrow K^{+} p \Lambda$ measurements 4 by assuming that the distributions in the $K^{+}$c.m. angle are isotropic. For clarity of presentation, the $3.30 \mathrm{GeV} / c$ data have been scaled by a factor of 0.6. The HIRES data at a beam momentum of $2.87 \mathrm{GeV} / c$ (inverted triangles) 6 were directly measured in an inclusive experiment. The vertical line indicates the position of the average $\Sigma^{+} n / \Sigma^{0} p$ threshold.

Also shown in Fig. 1 are the high resolution forward inclusive $K^{+}$production data from the HIRES collaboration 6 . These show a jump from the left to the right of the $\Sigma N$ threshold which has been interpreted as being primarily due to $\Sigma$ production 6 . However, since the exclusive COSY-TOF data show exactly the same kind of jump, though somewhat smaller in magnitude and smeared by resolution and binning, it is clear that a large fraction of what was assumed to be $\Sigma$ production corresponds in reality to the $p p \rightarrow K^{+} p \Lambda$ reaction 7 . 


\section{The $p p \rightarrow K^{+} n \Sigma^{+}$reaction}

Although there exist high energy bubble chamber data, the first modern $p p \rightarrow$ $K^{+} n \Sigma^{+}$experiment was carried out at COSY-11 by detecting the neutron in coincidence with the presumed $K^{+8}$. Remarkably large cross sections were found at excess energies of $Q=13$ and $60 \mathrm{MeV}$; these were about two orders of magnitude higher than those for $\Sigma^{0}$ production. The initial measurement at ANKE at $126 \mathrm{MeV}$ gave a much lower result 9 and it seems implausible that there could be an anomalous FSI to make these two data sets consistent. The $\Sigma^{0} p$ and $\Sigma^{+} n$ final states are both mixtures of isospin $I=1 / 2$ and $3 / 2$, and there is no unusual $\Sigma^{0} p$ behavior. In order to investigate this, further measurements were undertaken at ANKE at $Q=13,47,60$ and $82 \mathrm{MeV}^{10}$ and cross sections evaluated using three different techniques: (1) Study of inclusive $K^{+}$production, (2) Study of $K^{+} p$ coincidences, and (3) Study of $K^{+} \pi^{+}$coincidences. All three methods give consistent answers and show that the $\Sigma^{+}$production cross section is just a little less than that for $\Sigma^{0}$.

The most convincing of the inclusive measurements arises from looking at the ratio of $p p \rightarrow K^{+} X$ data taken $13 \mathrm{MeV}$ above the $\Sigma^{+} n$ threshold to those $5 \mathrm{MeV}$ below, since this does not depend upon the efficiency for $K^{+}$detection. The ANKE acceptance so close to threshold is quite high and the ratio shows that, at the $95 \%$ confidence level, the ratio of total production cross sections $\sigma\left(\Sigma^{+}\right) / \sigma\left(\Sigma^{0}\right) \lesssim 2$.

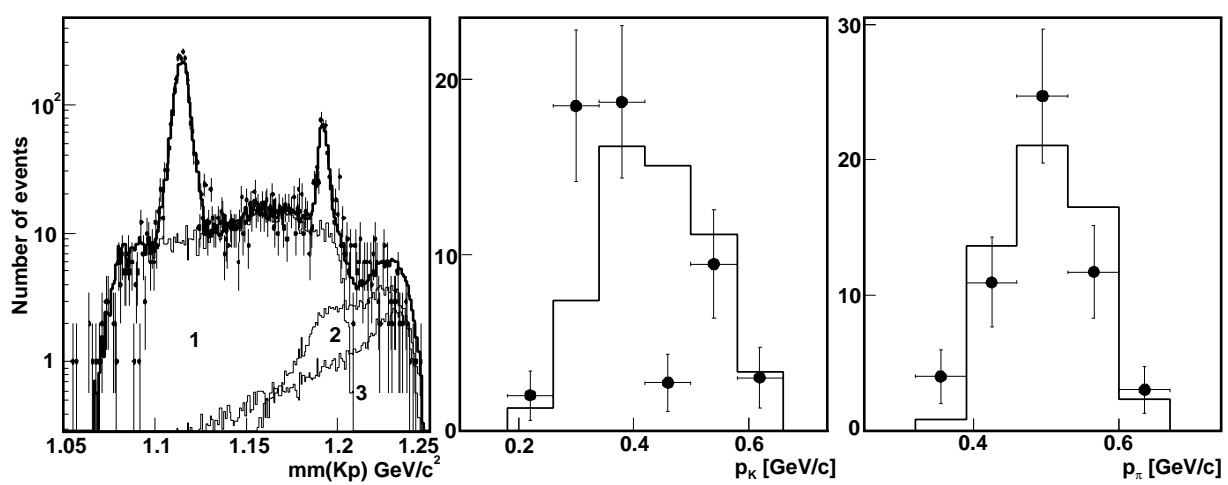

Fig. 2. Left: The $K^{+} p$ missing-mass spectrum at $1.958 \mathrm{GeV}$ 10. Apart from peaks corresponding to direct $\Lambda$ and $\Sigma^{0}$ production, there are also contributions where the proton has come from the decay of a $\Lambda(1)$, a $\Sigma^{0}(2)$, and a $\Sigma^{+}(3)$. Center and Right: $K^{+}$and $\pi^{+}$momentum spectra from $K^{+} \pi^{+}$coincidences at $1.958 \mathrm{GeV}$ compared to simulations 10 .

The results of the $K^{+} p$ coincidence measurements at $1.958 \mathrm{GeV}$ presented in Fig. 2 show peaks corresponding to $\Lambda$ and $\Sigma^{0}$ production, where the proton detected is the "direct" one. In addition there are indirect contributions where the proton comes from the decay of $\Lambda, \Sigma^{0}, \Sigma^{+}$. The simulations shown prove that, in the neighborhood of maximum missing masses, there can be no contamination from 
$\Lambda$ production and decay. If we then make the drastic assumption that the $\Sigma^{0}$ contributes nothing at all in this region, this gives $\sigma\left(\Sigma^{+}\right) / \sigma\left(\Sigma^{0}\right)<1-2$, depending upon energy.

Although only upper limits have so far been quoted, cross sections with error bars could be derived from the inclusive and $K^{+} p$ data. However, at low energies any $K^{+} \pi^{+}$coincidences detected in $p p$ collisions must come from $\Sigma^{+}$production. The $K^{+}$and $\pi^{+}$momentum spectra, obtained from these gold-plated events at $1.958 \mathrm{GeV}$ and shown in Fig. 2, are reasonably well described by the simulations. There are a few random coincidences that can be estimated from the below-threshold data. These data lead to cross section values that are completely consistent with the other two methods and show that $\sigma\left(\Sigma^{+}\right) / \sigma\left(\Sigma^{0}\right) \approx 0.7 \pm 0.1$, which is a long way below the HIRES assumption $[6$ and two orders below that of COSY-11 8 .

\section{The $\Sigma N$ final state interaction}

Although the energy dependence of the $p p \rightarrow K^{+} p \Sigma^{0}$ total cross section is inconsistent with a strong $\Sigma^{0} p$ FSI, it would be good to have independent confirmation. The potential for this is provided by the ANKE $p p \rightarrow K^{+} p X$ data 10 , an example of which is shown in Fig. 3. For the events in the $\Sigma^{0}$ peak in the left panel, the corresponding $\Sigma^{0} p$ invariant mass distribution in the right panel seems to be closer to phase space rather than one distorted by a FSI of $\Lambda p$ strength.
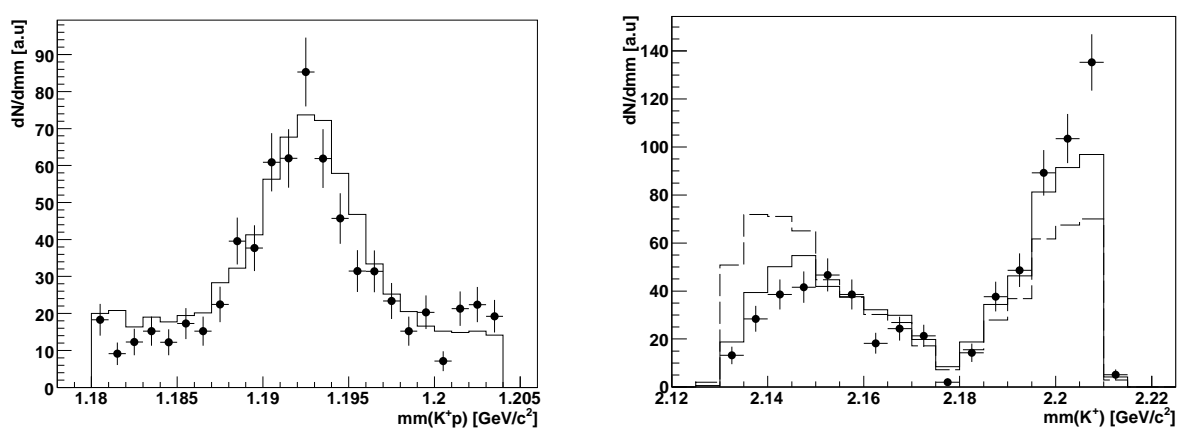

Fig. 3. Left: $K^{+} p$ missing mass from the $p p \rightarrow K^{+} p X$ reaction at $2.02 \mathrm{GeV} 10$ showing a prominent $\Sigma^{0}$ peak sitting on a physical background that is reproduced by simulation. Right: $K^{+}$missing mass, i.e. $\Sigma^{0} p$ invariant mass, for the events in the $\Sigma^{0}$ peak, compared to phase-space simulations (solid line) and these modified with a strongly attractive $\Sigma^{0} p$ FSI (dashed line).

Earlier experiments at COSY-TOF on $p p \rightarrow K^{0} p \Sigma^{+}$also showed no sign of an $I=3 / 2 \Sigma^{+} p$ FSI at 2.16 and $2.40 \mathrm{GeV}$, though the FSI region was comparatively small at these higher energies 11 . 


\section{Hyperon production in proton-neutron collisions}

All studies on the neutron have so far been undertaken in quasi-free kinematics using a deuterium target. The $p d \rightarrow p_{\mathrm{sp}} K^{0} p \Lambda$ reaction has been investigated at COSYTOF ${ }^{12}$ by detecting the combination of two delayed decays, viz. $K^{0} \rightarrow \pi^{+} \pi^{-}$and $\Lambda \rightarrow p \pi^{-}$, for which the apparatus is ideal. The momentum of the spectator proton, $p_{\mathrm{sp}}$, was then deduced through a kinematic fit. About 1000 fully reconstructed events are expected from the pilot run, though the c.m. energy has to be evaluated eventby-event and so the data are spread over a wide range.

A complementary approach has been proposed at ANKE 13 , where the spectator from $p d \rightarrow p_{\mathrm{sp}} K^{+}{ }_{n} \Lambda$ will be detected in telescopes placed in the target chamber. The spectator determines the c.m. energy and, below the $\Sigma$ thresholds, the $K^{+}$ signals the production of a $\Lambda$.

It should be noted that the total cross sections for $p p \rightarrow K^{+} p \Lambda, p n \rightarrow K^{0} p \Lambda$, and $p n \rightarrow K^{+} n \Lambda$ are linked by isospin, though there can be interferences between isospin- 0 and -1 in differential observables.

Data with direct spectator detection have already been taken at ANKE on $p d \rightarrow$ $p_{\mathrm{sp}} K^{+} p X^{14}$. The $p_{\mathrm{sp}} K^{+} p$ triple coincidence measurements resulted in about 500 $\Sigma^{-}$events at both 2.12 and $2.22 \mathrm{GeV}$.

\section{Comparison of $p p \rightarrow p K^{+} \Sigma^{0} \pi^{0}$ and $p p \rightarrow p K^{+} p K^{-}$reactions}

There have been measurements of both the $p p \rightarrow p K^{+} \Sigma^{0} \pi^{015}$ and $p p \rightarrow$ $p K^{+} p K^{-16}$ reactions at $2.83 \mathrm{GeV}$ at ANKE. Could these two associated production reactions be connected in some way? This might happen if the $\Sigma^{0} \pi^{0}$ and $p K^{-}$ were both produced through the decay of the $\Lambda(1405)$ resonance. What we would then be seeing is different manifestations of the reaction $p p \rightarrow p K^{+} \Lambda(1405)$.

In a new phenomenological meson-exchange mode $[17$, the reaction is assumed to proceed via the production and decay of the $N^{*}(1535) \rightarrow K^{+} \Lambda(1405)$, since it is believed that the $N^{*}(1535)$ isobar contains a lot of hidden strangeness. This assumption determines the strengths of the cross sections but not the shapes of the spectra shown in Fig. 4. These and the relative strengths are fixed mainly by low energy $\bar{K} p / \pi \Sigma$ data, which have been parametrized in terms of a separable potential 18 . The $K^{-} p$ predictions come out too high by about a factor of 2.6 , whereas the $\Sigma^{0} \pi^{0}$ are about right.

This factor of 2.6 between the quality of the two predictions depends very little at all on the $N^{*}(1535)$ assumptions; the effects of the low-energy coupled-channel physics dominate. Given the theoretical and experimental uncertainties, the plausible agreement here suggests that $K^{+} K^{-}$production in $p p$ collisions near threshold is also driven mainly by intermediate hyperon states and not, for example, by the production of scalar mesons.

In the time available I have chosen to highlight the COSY-TOF and COSYANKE contributions in "curiosity" production, they have also undertaken lots of non-strangeness studies. This talk must be for another day! 

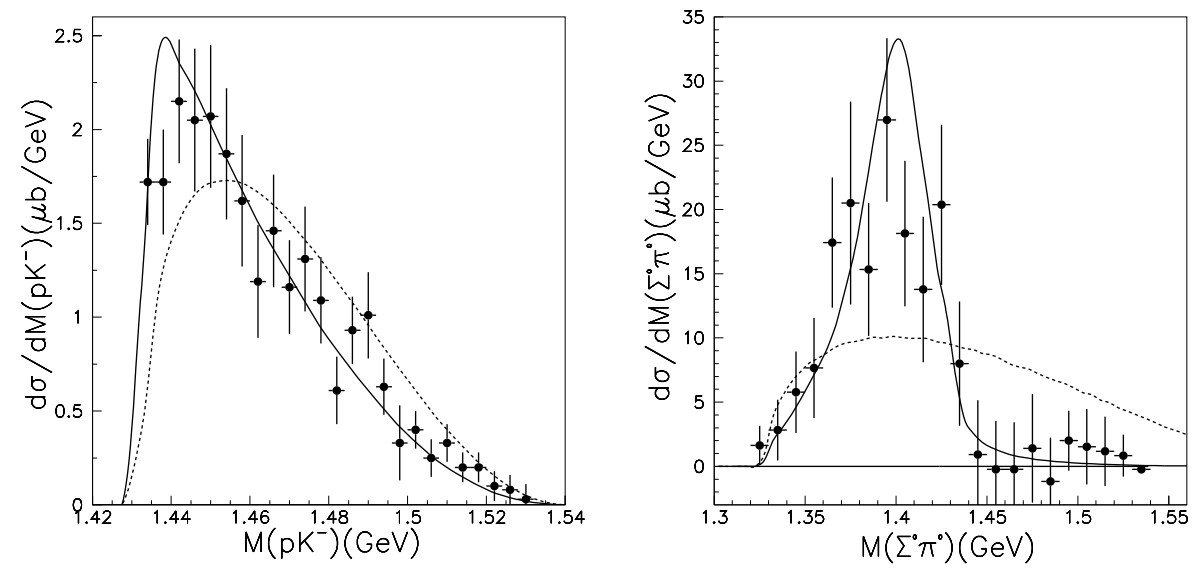

Fig. 4. Differential cross section for (Left) $p p \rightarrow p K^{+} K^{-} p$ at $Q=108 \mathrm{MeV} 16$ and (Right) $p p \rightarrow p K^{+} \pi^{0} \Sigma^{0}$ at $Q=212 \mathrm{MeV}$. The predictions of the $N^{*}(1535)$ mode 17 are shown by the solid lines which, in the $\Sigma^{0} \pi^{0}$ case, has been scaled down by a factor of 2.6. The dashed lines represent normalized four-body phase-space distributions.

\section{Acknowledgments}

Useful information on the COSY-TOF and COSY-ANKE experiments was supplied by W. Eyrich and Yu. Valdau. respectively. Support from the conference organizers is gratefully acknowledged.

\section{References}

1. http://www.fz-juelich.de/ikp/COSY-TOF/index e.html.

2. S. Barsov et al., Nucl. Instrum. Methods Phys. Res. A 462, 364 (2001).

3. H. A. Bethe and F. de Hoffmann, Mesons and Fields, vol. II Mesons (Row, Peterson \& Co., Evanston, 1955).

4. S. Abd El-Samad et al., Phys. Lett. B 688, 142 (2010).

5. S. Abd El-Samad et al., submitted to Eur. Phys. J. A.

6. A. Budzanowski et al., doi:10.1016/j.physletb.2010.07.015.

7. Y. Valdau and C. Wilkin (in preparation).

8. T. Rożek et al., Phys. Lett. B 643, 251 (2006).

9. Yu. Valdau et al., Phys. Lett. B 652, 245 (2007).

10. Yu. Valdau et al., Phys. Rev. C 81, 045208 (2010).

11. L. Karsch, PhD thesis, University of Dresden (2005).

12. M. Krapp, IKP-Jülich Annual Report (2009).

13. A. Dzyuba, V. Koptev and Yu. Valdau, COSY proposal \#203 (2010).

14. E. Shikov, Diploma thesis, University of St. Petersburg (2009).

15. I. Zychor et al., Phys. Lett. B 660, 167 (2008).

16. Y. Maeda et al., Phys. Rev. C 77, 015204 (2008).

17. Ju-Jun Xie and Colin Wilkin, Phys. Rev. C (in press), arXiv:1005.2957 (2010).

18. N. V. Shevchenko et al., Phys. Rev. Lett. 98, 082301 (2007). 